\title{
Application of the ensemble Kalman filter for assisted layered history matching
}

\author{
Wenshu Zha, Shanlu Gao*, Daolun Li, Kaijie Chen \\ Department of Mathematics, Hefei University of Technology, Hefei 230000, P. R. China
}

(Received October 5, 2018; Revised November 4, 2018; accepted November 6, 2018; available online November 12, 2018)

\section{Citation:}

Zha, W., Gao, S., Li, D., Chen, K.

Application of the ensemble Kalman filter

for assisted layered history matching.

Advances in Geo-Energy Research, 2018,

2(4): 450-456, doi:

10.26804/ager.2018.04.09.

Corresponding author:

*E-mail: shanlu.gao@ipp.ac.cn

Keywords:

Layered history matching

ensemble Kalman filter

normalization

production data

\begin{abstract}
:
Ensemble Kalman filter method has been used for automatic history matching the well production data such as production rate and watercut. However, the data of the connection watercut and connection rate are rarely used. In this work we conducted a history matching study based on the connection information using the EnKF for the first time to improve the matching accuracy. First, the initial implementation models are generated using the sequential Gaussian simulation method. Second, we choose the well watercut and connection watercut of each layer as production data respectively. During this step, the data such as permeability, pressure, saturation, and production data are normalized to improve the accuracy of history matching and reduce the simulation time. Finally, the case using the well watercut as historical production data is compared against the case using the connection watercut using EnKF. The results show that the well bottomhole pressure and connection watercut can be better matched using the connection watercut as the historical production data. In addition, the simulation time decreases significantly.
\end{abstract}

\section{Introduction}

Reservoir characterization is important in the petroleum industry to describe reservoir properties. The process of integrating dynamic data into the reservoir model to obtain reliable reservoir characterization is known as history matching (Oliver et al., 2008). Traditionally, history matching is aimed at adjusting the geological model and parameters manually using years of production data. These parameters including permeability and porosity are beneficial to reproduce the well flow rate and pressure histories reasonably (Li et al., 2003). One aims at minimizing the square of the mismatch between all measurements and computed values typically (Oliver and Chen, 2011). However, history matching such as the gradient method (Gao et al., 2017) and the genetic algorithm (Irani and Nasimi, 2011) is an inverse problem that requires complex and time-consuming computing.

From another perspective, the ensemble Kalman filter method based on the Bayesian approach is one approach of calibrating the reservoir models to save the high computational cost, which is one of the most efficient reservoir characterization methods.

Besides, the ensemble Kalman filter (EnKF) is a recursive data assimilation algorithm combining data with dynamical models to obtain the best estimation. It assimilates years of production data sequentially and updates static and dynamic parameters of reservoir model through the continuous use of observation data (Evensen, 2003). Optimal models are estimated based on matching the production data efficiently. It is with ease to combine EnKF with any forward simulator, and various model parameters can be adapted with EnKF. In the process of updating the reservoir model with the EnKF algorithm, reservoir properties can be estimated from the ensemble at any time step. The results are obtained by rerunning the reservoir simulator.

EnKF was presented for the first time by Evensen (Evensen, 1994) as a supplement to the classical Kalman filter (Kalman, 1960) about nonlinear problems. After that it has been widely used in the petroleum engineering related literature (Naevdal, 2002), EnKF has attracted a lot of research attention and has now been successfully applied to automatic history matching in the field (Haugen et al., 2008; Seiler et al., 2009), especially for the heterogeneous channel reservoirs (Jo et al., 2017; Jung et al., 2017).

Various conditions should be satisfied to be applied to the real field. As reservoirs become more complicated, iterative 
forms of EnKF (IEnKF) are presented to give better history matching results on the basis of EnKF (Krymskaya et al., 2009), Li and Reynolds (2009) also proposed IEnKF which need calculating the sensitivity matrix with the adjoint method, but it requires multiple assimilation steps to calibrate the models for obtaining optimal estimations. The ensemble size could be increased appropriately. There is no doubt that it increases time in operations. In addition, many other modified methods are presented to solve issues encountered, such as multiple Kalman gains (Lee et al., 2013), ensemble smoother (Kang et al., 2016), ensemble randomized maximum likelihood method (Chen and Oliver, 2012; Stordal and Naevdal, 2018), etc.

However, owing to the complex process of layered history matching, only a few literatures are concerned with layered history matching. Previous researches on history matching using EnKF mainly focused on the data measured in well. Most scholars use the data measured in wells rather than data combining connection information, such as connection watercut and connection rate. The problem hinders reliable reservoir estimation with lacking of connection information.

In this work, we introduced the data of connection watercut to observed data and conduct the layered history matching using the EnKF method, which improves the accuracy of the matching results. Furthermore, we proposed to process the state vector with normalization before the EnKF iteration to reduce the simulation time.

\section{Methodology}

\subsection{Ensemble Kalman filter method}

EnKF method is the calibration of the traditional Kalman filter. Eisenmenn (Eisenmenn et al., 1994) tried to apply the traditional Kalman filter to reservoir characterization problems which is limited to small number of parameters and only when there is linear relationship between the observation data and model parameters (Corser et al., 2000). The main difference with EnKF is to use the statistics of a succession of ensembles to obtain the covariance matrix.

EnKF is an effective data assimilation algorithm to estimate unknown parameters in high-dimensional systems (Luo et al., 2011), and the reservoir production estimations are improved sequentially after data are assimilated. The EnKF method aims at performing numerical calculations to update the reservoir dynamic properties (Jung et al., 2018). We define the state vector for the model data. The state vector consists of reservoir static model parameters $m$, reservoir dynamic parameters $p^{n}$ and the predicted data $d^{n}$ at time $t_{n}$ as described in the following equation:

$$
y_{n, j}=\left(\begin{array}{l}
m \\
p^{n} \\
d^{n}
\end{array}\right), j=1, \cdots, N_{e}
$$

where $m$ is a $N_{m}$ dimensional column vector including porosity and permeability which is time independent; $p^{n}$ is a $N_{p}$ dimensional column vector, including pressure, saturation, dissolved gas-oil ratio, etc, which is time dependent during the production process. It is usually the solution to the flow equation; $d^{n}$ is a $N_{d}$ dimensional column vector which represents the predicted data related to the geologic model and dynamic field, including well bottomhole pressure, well watercut, etc. The subscript $j$ represents the $j^{\text {th }}$ implementation of the whole ensemble. $y_{n, j}$ is the state vector of the $j^{t h}$ implementation at time $t_{n}$.

In EnKF, the first step is to forecast. $F$ is the forward simulator. In forecast step, only the dynamic parameters and predicted data are changed with time, and the static parameters remain unchanged which is shown in Eq. (3). Utilizing given present static parameters, the state vector to the next time step is calculated as in Eq. (2).

$$
\begin{gathered}
y_{n, j}^{f}=F\left(y_{n-1, j}^{u}\right) \\
m_{n, j}^{f}=m_{n-1, j}^{u}
\end{gathered}
$$

The observation equation is:

$$
\begin{gathered}
H=\left[\begin{array}{ll}
O & I
\end{array}\right] \\
d_{o b s}^{n}=H y_{n}+v(n)
\end{gathered}
$$

$H$ is the measurement operator, consisting of only 0 and 1 . $O$ represents $N_{d} \times\left(N_{m}+N_{p}\right)$ null matrix. $I$ represents $N_{d} \times N_{d}$ identity matrix. It is assumed that $v(n)$ is the meaurement error obeying the Gaussian distribution with zero mean and covariance matrix given by $C_{D n}$.

Using Bayes' theorem, the probability density function (pdf) for $y$ conditional to $d_{o b s}$ is given by (Zafari and Reynolds, 2005).

$$
\begin{aligned}
f\left(y_{n} \mid d_{o b s}^{n}\right) \propto \exp [ & -\frac{1}{2}\left(y_{n}-\overline{y_{n}}\right)^{T} C_{y, n}^{-1}\left(y_{n}-\overline{y_{n}}\right) \\
& \left.-\frac{1}{2}\left(H y_{n}-d_{o b s}^{n}\right)^{T} C_{D n}^{-1}\left(H y_{n}-d_{o b s}^{n}\right)\right]
\end{aligned}
$$

By sampling the pdf, EnKF generates a succession of implementations of the data by adding noise. For any $j$,

$$
d_{u c, j}^{n}=d_{o b s}^{n}+L_{D} Z_{D}
$$

where $C_{D n}=L_{D} L_{D}^{T}$ is the Cholesky decomposition of $C_{D n}$. $Z_{D}$ is the normal random vector with zero mean and covariance $I_{N_{d}}$ given by identity matrix.

We defined the following objective function from the conditional probability density in Eq. (6).

$$
\begin{aligned}
O\left(y_{n, j}\right)= & \frac{1}{2}\left(y_{n, j}-y_{n, j}^{f}\right)^{T} C_{y, n}^{-1}\left(y_{n, j}-y_{n, j}^{f}\right) \\
& +\frac{1}{2}\left(H y_{n, j}-d_{u c, j}^{n}\right)^{T} C_{D n}^{-1}\left(H y_{n, j}-d_{u c, j}^{n}\right)
\end{aligned}
$$

In the assimilation step, $y_{n, j}^{f}$ represents the $j^{\text {th }}$ forecasted state vector at time $t_{n}$. We can get standard EnKF analysis equation by minimizing the objective function $O\left(y_{n, j}\right)$. The 


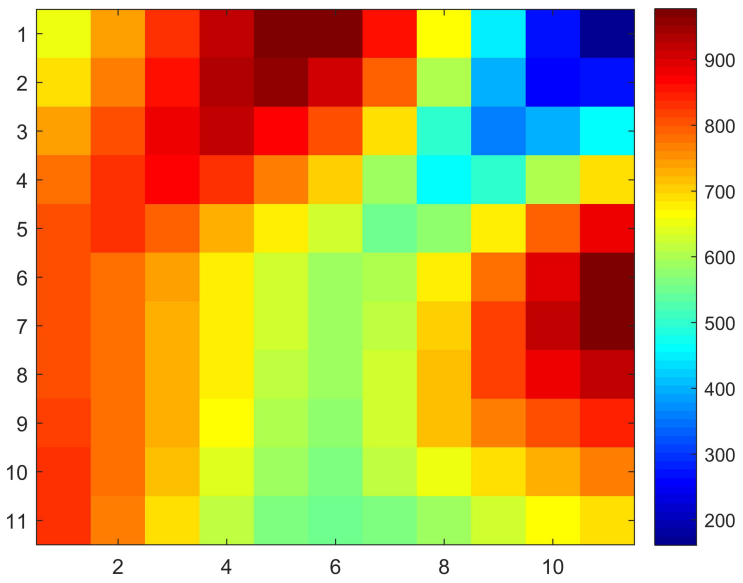

Fig. 1. Real permeability field.

state vectors are updated as Eq. (9) with Kalman filter gain $K_{n}$ (Eq. (10)).

$$
\begin{gathered}
y_{n, j}^{u}=y_{n, j}^{f}+K_{n}\left(d_{u c, j}^{n}-H y_{n, j}^{f}\right) \\
K_{n}=C_{y, n}^{f} H^{T}\left(H C_{y, n}^{f} H^{T}+C_{D n}\right)^{-1}
\end{gathered}
$$

$C_{y, n}^{f}$ is the covariance matrix of state vector, $\overline{Y_{n}^{f}}$ is the average of state vector. Superscript of $u$ and $f$ denote 'updated' and 'forecasted', respectively.

$$
C_{y, n}^{f}=\frac{1}{N_{e}-1}\left(Y_{n}^{f}-\overline{Y_{n}^{f}}\right)\left(Y_{n}^{f}-\overline{Y_{n}^{f}}\right)^{T}
$$

The covariance matrix $C_{y, n}^{f}$ can also be updated with the following equation:

$$
C_{y, n}^{u}=\left(I-K_{n} H\right) C_{y, n}^{f}
$$

\subsection{Normalization}

Standarlization of data is to scale the data to a specific interval. It is widely used for purposes of comparison and evaluation. To facilitate the comparison and evaluation of data, we eliminated the limitations of different units or magnitude by transforming data into nondimensional value during the process of data standardization.

The most typical one is the normalization of data, which is to map the data to the interval of $[0,1]$ uniformly. The method we used in this work is min-max scaling, the formula of normalization is:

$$
X_{\text {norm }}=\frac{X-X_{\min }}{X_{\max }-X_{\min }}
$$

where $X$ is the initial sample data. $X_{\max }, X_{\min }$ are the maximum and minimum of $X$ respectively, and $X_{\text {norm }}$ is the normalization data. Then, the value can be returned with the following Eq. (14) after each iteration.

$$
X=X_{\text {norm }}\left(X_{\max }-X_{\text {min }}\right)+X_{\text {min }}
$$

In this work, we processed the state vector with normalization during the iteration of the EnKF loop. The results are improved because of eliminating the big difference of each layer.

\section{Experiments}

\subsection{Application of EnKF updating}

We applied the EnKF algorithm to a reservoir model to match the history production data. The reservoir model consists of $11 \times 11 \times 3$ grid blocks with two phases of oil and water. The size of each gridblock is $30 \mathrm{~m} \times 25 \mathrm{~m} \times 20 \mathrm{~m}$. Four producers and one injector are exerted in this reservoir. In order to combine layered history matching with EnKF, we utilized EnKF to update permeability on the ensemble models and match the production data, including well watercut and connection watercut. The permeability field was estimated by matching production data above. Their performances were compared among well bottomhole pressure (WBHP), well watercut (WWCT), connection watercut (CWCT). We generated 100 initial models by using sequential Gaussian simulation (SGS). Just several points were used from the data, so there are considerable uncertainties between the initial models and true field.

We took permeability data on the production wells as input reservoir information. Take second layer of the reservoir for example. The real permeability field is shown in Fig. 1. Four models of the initial ensemble are listed which is shown in Fig. 2. The average over the propagated ensemble models is displayed in Fig. 3.

The production time lasts for 25 years that the state vector is updated every month by choosing the monthly WWCT and CWCT as production data. Two cases of EnKF updates were 


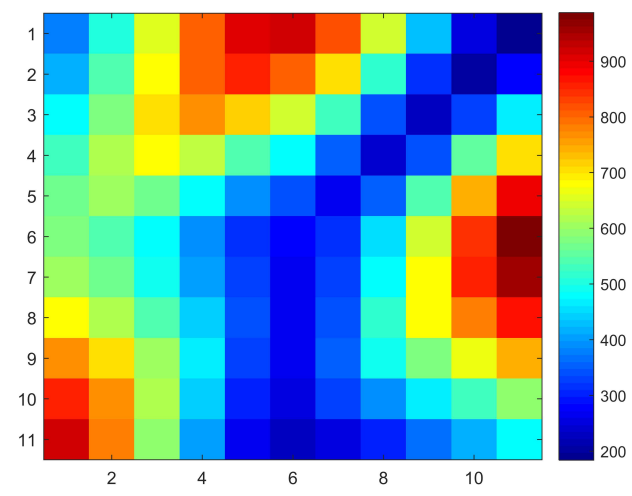

(a)

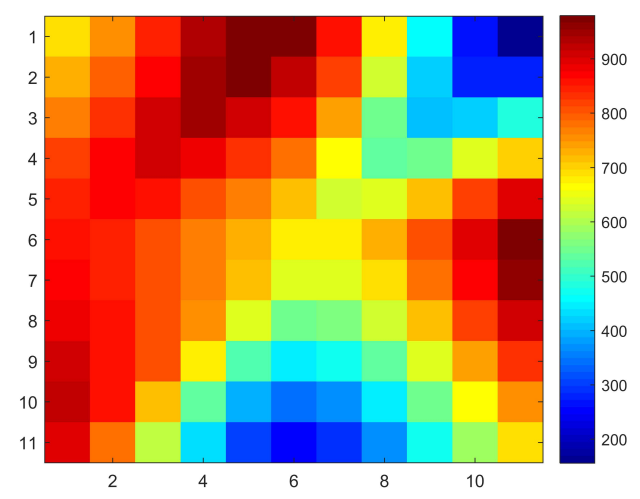

(c)

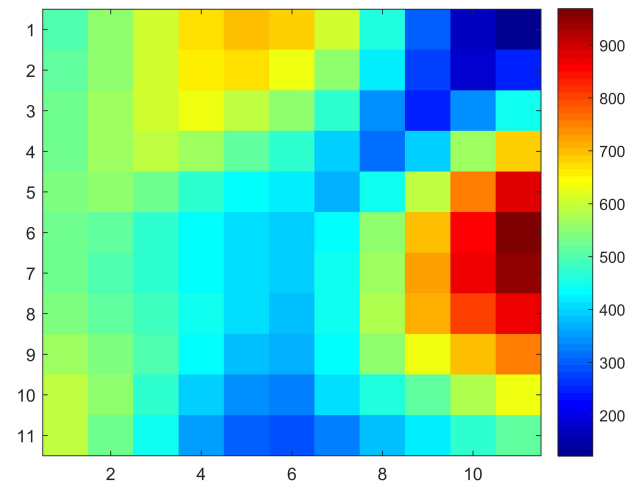

(b)

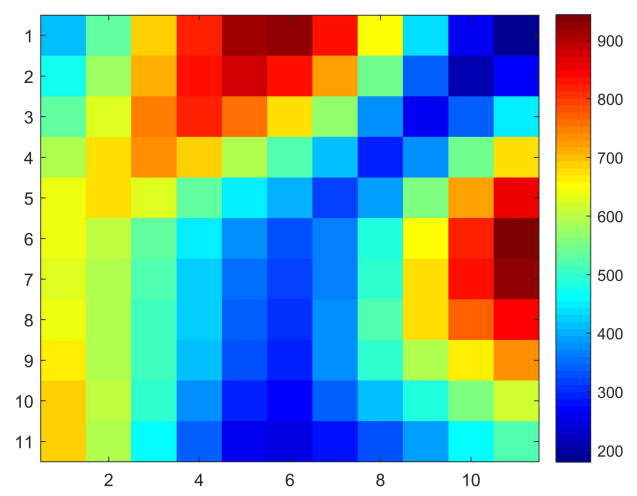

(d)

Fig. 2. Four models of the initial ensembles.

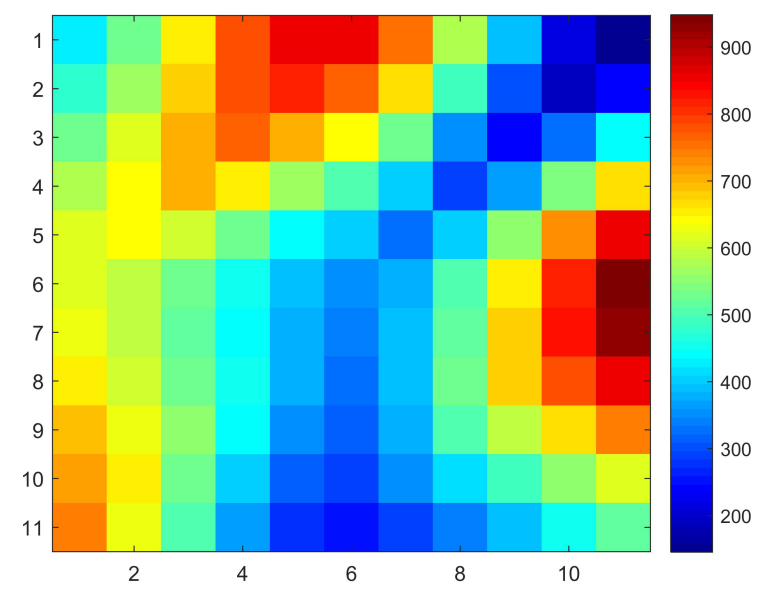

Fig. 3. Average prior model. 


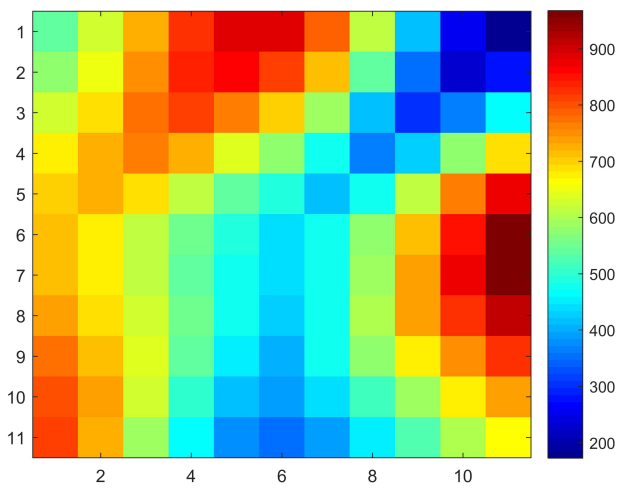

(a)

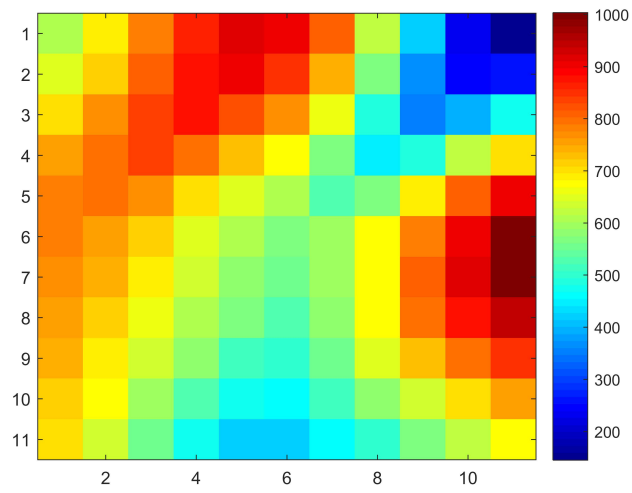

(b)

Fig. 4. The permeability field after EnKF updates. (a) Case 1 after EnKF updates; (b) Case 2 after EnKF updates.

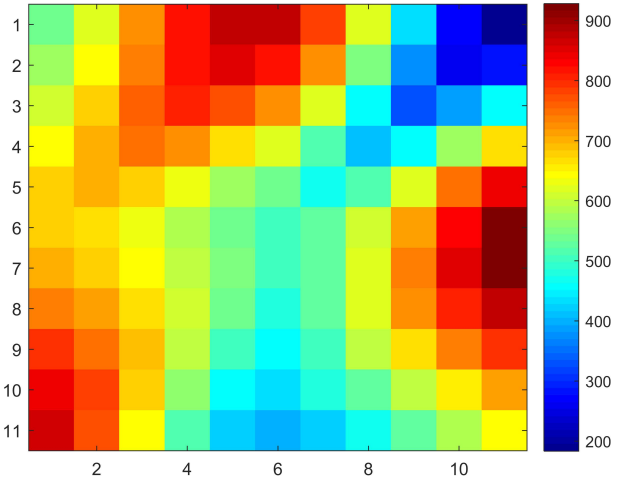

(a)

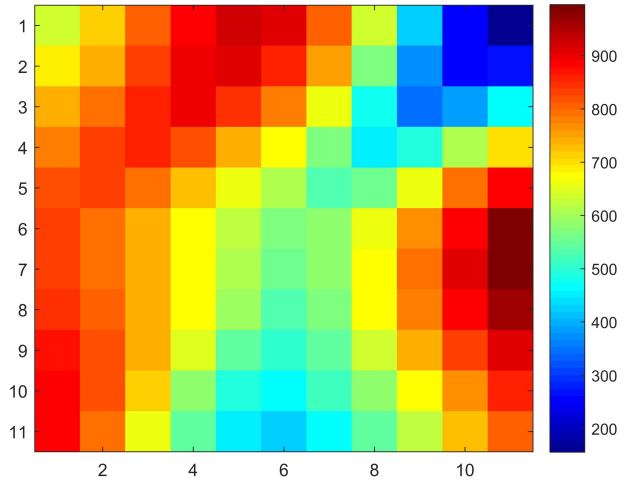

(b)

Fig. 5. EnKF updates with normalization. (a) EnKF of case 1 with normalization; (b) EnKF of case 2 with normalization.

run for comparison: i) In case 1, we choose the monthly WWCT as the production data; ii) we choose the CWCT as the production data considering layered information in case 2. Meanwhile, we compared the permeability fields of case 1 and case 2 as in Fig. 4.

Compared with the average prior model (shown in Fig. 3), we found that the updated permeability fields of case 1 (shown in Fig. 4(a)) and case 2 (shown in Fig. 4(b)) are both similar to the real permeability field. However, the latter provides a better estimation of the permeability field because layered information is included.

\subsection{Improvement with normalization}

To eliminate the large difference between different layers and reduce the simulation time, we normalized the state vector according to each category during the iteration of EnKF. Then the value is returned after each iteration according to Eq. (14) as the input value in the reservoir simulator.

We normalized the data of case 1 and case 2 before EnKF updates, according to each category of model parameters respectively. EnKF updates with normalization are shown in Fig.
5. Compared with the real permeability field, the permeability fields of both cases are improved with normalization.

In addition, Fig. 5(b), which is the result after two iterations, shows that the values of some grid points are closer to the real values compared to the estimation result after eight iterations in Fig. 5(a). Therefore, though case 2 utilizes the same ensemble as case 1, the simulation time is cut by $75 \%$ and a better result is obtained.

\subsection{Comparison of two cases}

We compared the matching results of both cases of EnKF updating with normalization. Fig. 6 compares two matching results of WBHP in two cases. Case 2 is close to the true value in the early days, and as more and more data are assimilated, it matches the true value better after approximately 6,000 days, which proves that the matching result in case 2 is more accurate. Fig. 7 compares fitting results of WWCT in two cases. Case 2 achieves better goodness of fit than case 1. Fig. 8 compares fitting results of CWCT in each layer. Although the goodness of fit in the first layer is not as good as that of the second and third layer, the value of case 2 is always closer 


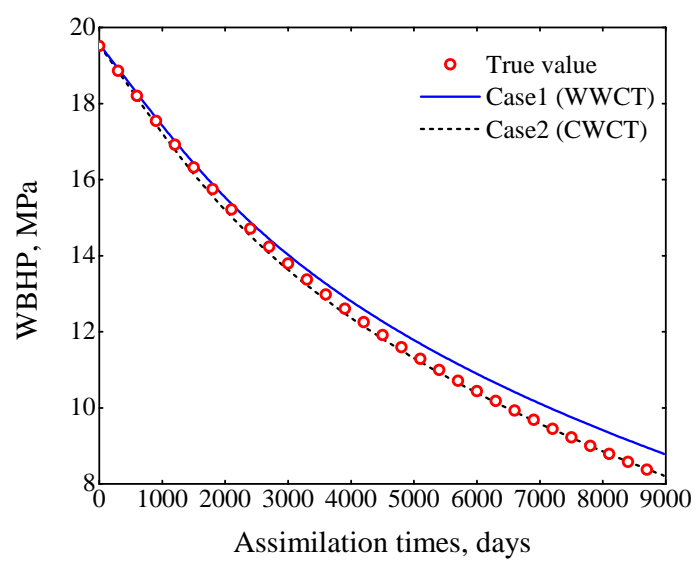

Fig. 6. Comparison of fitting results of WBHP in two cases.

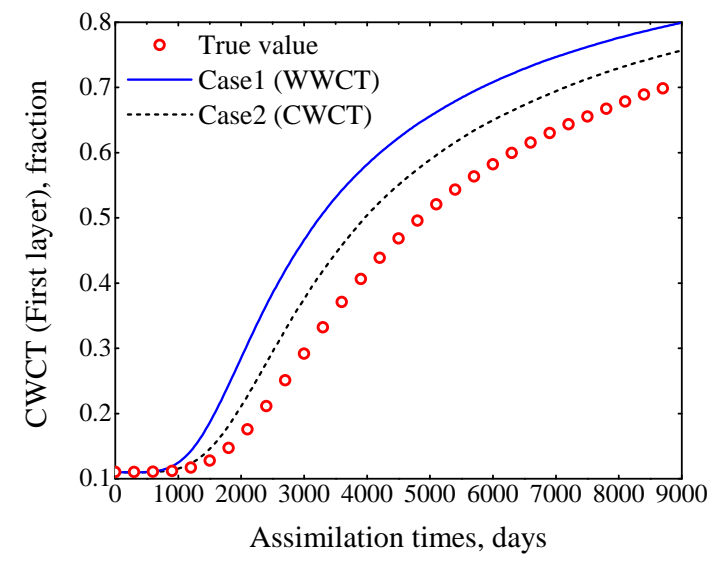

(a)

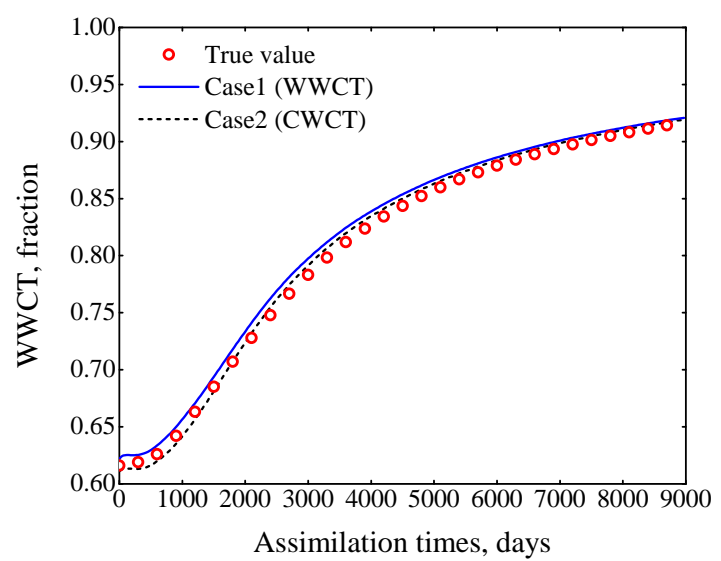

Fig. 7. Comparison of fitting results of WWCT in two cases.

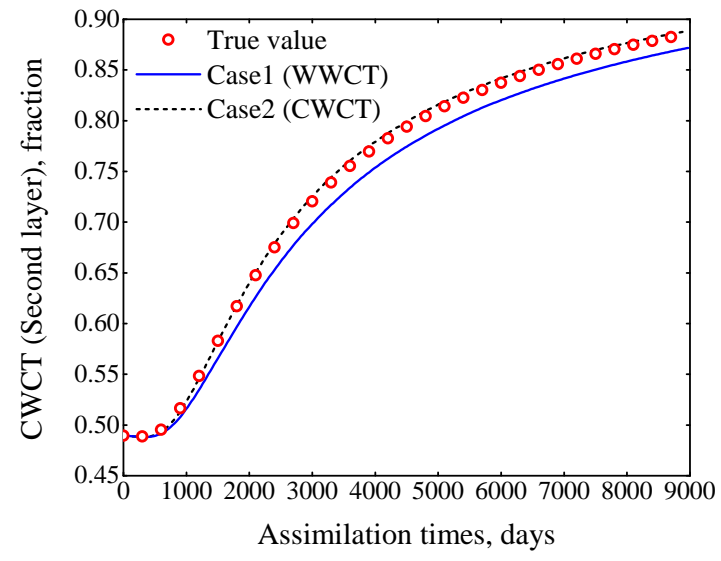

(b)

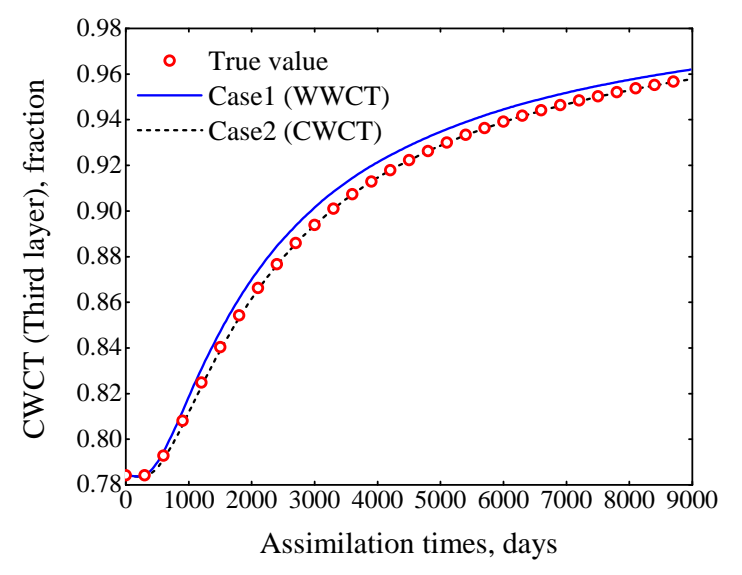

(c)

Fig. 8. Comparison of fitting results of CWCT in two cases. (a) CWCT of the first layer; (b) CWCT of the second layer; (c) CWCT of the third layer. 
to the true value than case 1 . On the whole, it provides better results in the second and third layers, especially in the third layer. Case 2 combining connection information shows better results on matching results than case 1 .

\section{Conclusions}

In this work, we introduced the layered history matching into EnKF, compared the difference between the well watercut and connection watercut as historical production data. Results show that the latter improves the accuracy of matching in each layer. Layered history matching utilizing EnKF provides more accurate results. Also, EnKF with normalization is valid for the model to update and perform the history matching. Meanwhile, it reduces the simulation time up to $75 \%$.

\section{Acknowledgments}

This work was sponsored by grants from CAS Strategic Priority Research Program (XDB10030402) and National Key Science and Technology Project (2017ZX05009005-002).

Open Access This article is distributed under the terms and conditions of the Creative Commons Attribution (CC BY-NC-ND) license, which permits unrestricted use, distribution, and reproduction in any medium, provided the original work is properly cited.

\section{References}

Chen, Y., Oliver, D.S. Ensemble randomized maximum likelihood method as an iterative ensemble smoother. Math. Geosci. 2012, 44(1): 1-26.

Corser, G.P., Harmse, J.E., Corser, B.A., et al. Field test results for a real-time intelligent drilling monitor. Paper SPE 59227 Presented at the SPE Drilling Conference, New Orleans, Louisiana, 23-25 February, 2000.

Eisenmann, P., Gounot, M.T., Juchereau, B., et al. Improved Rxo measurements through semi-active focusing. Paper SPE 28437 Presented at the SPE Annual Technical Conference and Exhibition, New Orleans, Louisiana, 25-28 September, 1994.

Evensen, G. Sequential data assimilation with a nonlinear quasi-geostrophic model using Monte Carlo methods to forecast error statistics. J. Geophys. Res. 1994, 99(C5): 10143-10162.

Evensen, G. The ensemble Kalman filter: Theoretical formulation and practical implementation. Ocean Dyn. 2003, 53(4): 343-367.

Gao, G., Vink, J.C., Chen, C., et al. Distributed Gauss-Newton optimization method for history matching problems with multiple best matches. Comput. Geosci. 2017, 21(5-6): 1325-1342.

Haugen, V., Naevdal, G., Natvik, L.J., et al. History matching using the ensemble Kalman filter on a North Sea field case. SPE J. 2008, 13(4): 382-391.

Irani, R., Nasimi, R. Evolving neural network using real coded genetic algorithm for permeability estimation of the reservoir. Expert Syst. Appl. 2011, 38(8): 9862-9866.

Jo, H., Jung, H., Ahn, J., et al. History matching of channel reservoirs using ensemble Kalman filter with continuous update of channel information. Energy Explor. Exploit. 2017, 35(1): 3-23.

Jung, H., Jo, H., Kim, S., et al. Recursive update of channel information for reliable history matching of channel reservoirs using EnKF with DCT. J. Pet. Sci. Eng. 2017, 154: 19-37.

Jung, S., Lee, K., Park, C., et al. Ensemble-based data assimilation in reservoir characterization: A review. Energies 2018, 11(2): 445.

Kalman, R.E. A new approach to linear filtering and prediction problems. Trans. ASME J. Basic Eng. 1960, 82(1): 35-45.

Kang, B., Lee, K., Choe, J. Improvement of ensemble smoother with SVD-assisted sampling scheme. J. Pet. Sci. Eng. 2016, 141: 114-124.

Krymskaya, M.V., Hanea, R.G., Verlaan, M. An iterative ensemble Kalman filter for reservoir engineering applications. Comput. Geosci. 2009, 13(2): 235-244.

Lee, K., Jeong, H., Jung, S.P., et al. Improvement of ensemble smoother with clustered covariance for channelized reservoirs. Energy Explor. Exploit. 2013, 31(5): 713726.

Lee, K., Jung, S.P., Shin, H., et al. Uncertainty quantification of channelized reservoir using ensemble smoother with selective measurement data. Energy Explor. Exploit. 2014, 32(5): 805-816.

Li, R., Reynolds, A.C., Oliver, D.S. History matching of threephase flow production data. SPE J. 2003, 8(4): 328-340.

Luo, X., Hoteit, I., Duan, L., et al. Review of nonlinear Kalman, ensemble and particle filtering with application to the reservoir history matching problem. Int. J. Urol. 2011, 21(1): 108-112.

Naevdal, G., Mannseth, T., Vefring, E. Near-well reservoir monitoring through ensemble Kalman filter. Paper SPE 75235 Presented at the SPE/DOE Improved Oil Recovery Symposium, Tulsa, Oklahoma, 13-17 April, 2002.

Oliver, D.S., Chen, Y. Recent progress on reservoir history matching: A review. Comput. Geosci. 2011, 15(1): 185221.

Oliver, D.S., Reynolds, A.C., Liu, N. Inverse Theory for Petroleum Reservoir Characterization and History Matching. Cambridge, UK, Cambridge University Press, 2008.

Seiler, A., Evensen, G., Skjervheim, J.A. Advanced reservoir management workflow using an EnKF based assisted history matching method. Paper SPE 118906 Presented at the SPE Reservoir Simulation Symposium, Woodlands, Texas, 2-4 February, 2009.

Stordal, A.S., Naevdal, G. A modified randomized maximum likelihood for improved Bayesian history matching. Comput. Geosci. 2018, 22(1): 29-41.

Zafari, M., Reynolds, A.C. Assessing the uncertainty in reservoir description and performance predictions with the ensemble Kalman filter. Paper SPE 95750 Presented at the SPE Annual Technical Conference and Exhibition, Dallas, Texas, 9-12 October, 2005. 